\title{
SISTEMA FOTOVOLTAICO CONECTADO A LA RED DE ENERGÍA ELÉCTRICA DEL DEPARTAMENTO DE INGENIERÍA ELECTROMECÁNICA DE LA UNNE
}

L. Vera ${ }^{1}$, F Gonzalez $^{2}$, A. Firman ${ }^{1}$, M Cáceres $^{1}$, A Busso $^{1}$

\section{RESUMEN}

En el presente trabajo se exponenla integración realizadas en el diseño y los resultados del monitoreo de un patio urbano que cuenta con un SFCR de $2,8 \mathrm{kWp}$, ubicado en el Campus de la Reforma Universitaria de la Universidad Nacional del Nordeste, cita en la ciudad de Resistencia

El diseño del patio urbano fue elaborado sobre un abordaje en el que se propone un espacio intermedio entre el campus y la ciudad, que permite vincular a los alumnos y a la ciudadanía a través de un espacio común, que incorpora una "cubierta solar" que actúa como lugar de encuentro y acercamiento con la tecnología fotovoltaica.

En el diseño se incorporan criterios de sustentabilidad ambiental como ser la reutilización de troncos acopiados en el terreno, la preservación de la "permeabilidad del suelo", así como la concreción de un nodo experimental que permita monitorear los resultados de este proyecto demostrativo.

La cubierta solar tiene un importante "efecto demostrativo" tendiente a la difusión de la energía fotovoltaica (limpia, renovable y sustentable), mediante la incorporación del concepto de coexistencia de los sistemas tradicionales de generación y distribución de energía eléctrica, en un espacio útil para el esparcimiento y actividades académicas a cielo abierto, iniciativa que lo que representa un cambio conceptual.

De esta manera, se plantea una experiencia aplicada que contribuirá al conocimiento tecnológico necesario para impulsar un cambio de escala en el uso de la energía solar, mediante un trabajo interdisciplinario de distintas áreas de la Universidad de las Facultades de Arquitectura, Ingeniería y Ciencias Exactas. Resultados obtenidos presentan al sistemas con índices de desempeño globales cuyos valores coinciden los con presentados por otros autores.

GER - Grupo en Energías Renovables - FaCENA - UNNE. Av. Libertad 5470 - 3400 Corrientes. Argentina.

Tel./Fax: (0379) 4473931 int 116, e-mail: luis.horacio.vera@comunidad.unne.edu.ar

${ }^{1}$ Investigador Grupo en Energías Renovables - UNNE.

${ }^{2}$ Becario Grupo en Energías Renovables - UNNE. 
Palabras Claves: Energía Solar, Sistemas Fotovoltaicos Conectados a la Red, Integración Urbana De Sistemas De Generación Eléctrica.

\section{INTRODUCCIÓN:}

$\mathrm{El}$ aumento en el precio de los recursos energéticos fósiles, la disminución de las reservas existentes, los problemas ambientales asociados y la demanda mundial de energía que crece continuamente ha conducido al sistema energético mundial, que se afianzó sobre una base centralizada de fuentes finitas no renovables, a cambiar este paradigma a través de sistemas de abastecimiento descentralizados de energía que utilizan recursos renovables. En una de las vertientes de este nuevo modelo se encuentra la tecnología solar de generación distribuida integrada a entornos urbanos.

El mercado fotovoltaico (FV) mundial ha tenido un fuerte crecimiento durante los últimos años como consecuencia de las políticas de promoción implementadas por diversos países, que han impulsado la instalación de sistemas $\mathrm{FV}$ conectados a la red de distribución eléctrica. Esto ha producido un nuevo récord de capacidad instalada durante 2015, con unos $50 \mathrm{GW}$ a nivel global, totalizando una capacidad de 227 GW (REN21, 2016). Las Figuras 1 y 2 muestran, respectivamente, la evolución histórica del mercado FV mundial en el período 1995-2015, y los países con mayor potencia FV instalada a fines de 2015.

En algunos países, la generación solar FV tiene un rol importante $\mathrm{Si}$ bien hasta recientemente la demanda de sistemas FV estuvo concentrada en los países desarrollados, en la actualidad los mercados emergentes al crecimiento global, particularmente en países en desarrollo.

Como consecuencia del crecimiento descripto, los precios internacionales de los sistemas FV y especialmente de los módulos $\mathrm{FV}$, han tenido una baja muy significativa en los últimos años, llevando el costo de la generación $\mathrm{FV}$ a valores prácticamente competitivos con la generación convencional en una cantidad importante de mercados, estimándose que la paridad con la red se alcanzará en la mayoría de los países durante la corriente década (Laborde y Williams 2016).

Por otra parte, la generación FV distribuida1 ha ido ganando mercado, muy especialmente en países europeos donde ha tenido una posición predominante. Más allá de sus ventajas comparativas con respecto a la generación centralizada en cuanto a ladisminución de pérdidas de energía por transmisión. A nivel global, se estima que en 2016 la generación distribuida (instalaciones de menos de $5 \mathrm{MW}$, en sectores residenciales, comerciales e industriales) tendrá una participación del $39 \%$ del total de la generación eléctrica FV conectada a la red (Munsell, 2016).

La situación en la Argentina es significativamente diferente, siendo la contribución de las energías renovables, excluyendo la generación hidroeléctrica de gran escala, muy pequeña. la participación de la energía eólica y solar en la matriz eléctrica fue de sólo $0,44 \%$, provista, en su gran mayoría, por energía eólica (CAMMESA, 2016). Resulta, en consecuencia, imprescindible promover la diversificación de la matriz energética, en particular mediante la introducción gradual de otras fuentes renovables de energía como la solar y eólica.

Por el contrario, no existen a nivel na- 
cional regulaciones técnicas ni políticas de promoción que permitan e impulsen la instalación de sistemas $\mathrm{FV}$ conectados a las redes de baja tensión. Con el objeto de promover en el país la generación de electricidad mediante sistemas $\mathrm{FV}$ de pequeñas potencias (típicamente, entre $2 \mathrm{kWp}$ y 50 $\mathrm{kWp}$ ), integrados a edificios y conectados a la red eléctrica de baja tensión, el Consorcio Público-Privado IRESUD, conformado por la CNEA, la UNSAM y 5 empresas privadas, ejecutó, entre diciembre de 2011 y abril de 2016, el proyecto "Interconexión de sistemas fotovoltaicos a la red eléctrica en ambientes urbanos", en adelante, proyecto IRESUD (IRESUD, 2016). Este proyecto estuvo parcialmente subsidiado con Fondos Argentinos Sectoriales (FITS Energía Solar No 0008-2010) del Ministerio de Ciencia, Tecnología e Innovación Productiva.

A nivel mundial, los países desarrollados, ya cuentan en su matriz energética con el aporte de la generación descentralizada a través de sistemas fotovoltaicos conectados a la red (SFCR). Actualmente, en la República Argentina, se está implementando esta nueva política energética a través de la realización de los primeros SFCR experimentales demostrativos en entornos urbanos. Dentro de estas iniciativas, una de la más importante es llevada adelante por el FONARSEC a través de la convocatoria FITS 2010 - Energía Solar, que financia parcialmente el Proyecto "Interconexión de Sistemas Fotovoltaicos a la Red Eléctrica en Ambientes Urbanos". Proyecto en el cual participa la Universidad Nacional del Nordeste realizando su aporte en el diseño, instalación y seguimiento de siete SFCR en provincias del Norte argentino.

En este trabajo se presentan las características constructivas, técnicas y resultados de funcionamiento de un SFVCR de 2,8KWp, ubicado en el Campus de la Reforma Universitaria de la universidad antes nombrada, en la ciudad de Resistencia. Instalación realizada dentro de la orbita del proyecto "Interconexión de Sistemas Fotovoltaicos a la Red Eléctrica en Ambientes Urbanos".

\section{ASPECTOS TECNOLÓGICOS}

La elaboración de un proyecto arquitectónico y técnico de un SFCR requiere una serie de análisis previos que proporcionen a la instalación, luego de su implementación, confiabilidad, seguridad, garantía de una generación adecuada de energía, así como un adecuado diseño que se ajuste a las condiciones del entorno. Un análisis de los posibles lugares de implantación del SFCR es fundamental ya que permite al proyectista determinar el tipo y lugar más adecuado. Otras variables necesarias a considerar fueron que el sitio estuviera lo suficientemente despejado para que la incidencia solar sea plena y directa; que pudiera ubicarse en bordes urbanos en contacto con la trama de la ciudad; en un sitio de visibilidad para la promoción de la experiencia por un lado y el cuidado del equipamiento a partir del control de la mirada colectiva, entre otros aspectos. Estas consideraciones, sumadas al hecho de la necesidad de control y monitoreo del sistema, fueron las razones que condujeron a la instalación del SFCR en un predio del Campus de la UNNE, cercano al departamento de Electricidad de la facultad de Ingeniería.

En las Fig. 1 a) puede apreciarse el lugar físico de implementación del SFCR, cuyas coordenadas son Latitud $27^{\circ} 28^{\prime} 2.86^{\prime \prime} \mathrm{S}$, Longitud 58 58'57.15”O, en la intersección de la Avenida Castelli y la Calle Dr. Ramírez, perteneciente 
al Campus de la Reforma, lindante con el edificio que alberga el área de Mecánica de la Facultad de Ingeniería, donde se realizara la implantación física del SFCR.

Desde el punto de vista constructivo la propuesta se basa en criterios de racionalidad, modularidad y economía. La cubierta está conformada por un arreglo de doce pa- neles fotovoltaicos. El arreglo se encuentra apoyado sobre una estructura de perfiles de chapa en voladizo. Los apoyos se materializan reutilizando troncos acopiados en el terreno, a modo de columnas apareadas. Este "gesto" estético se reitera en el diseño de una escultura a modo de hito que "atraviesa" el límite del campus como metáfora de la apertura de la universidad a su medio(Fig. 1 b).

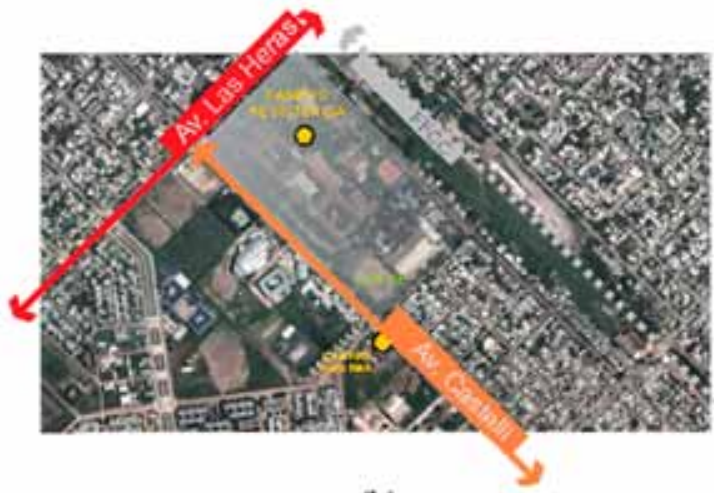

(b)

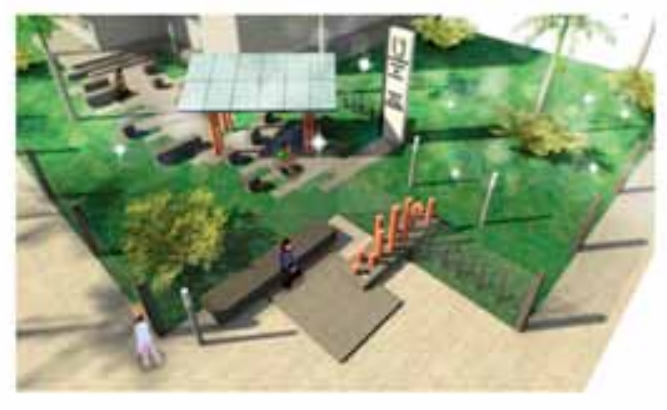

(a)

Figura 1, a) Implantación del Campus Resistencia y Campus de la Reforma Universitaria de la Universidad Nacional del Nordeste en la trama urbana de la ciudad de Resistencia.

Fuente: Imagen de Google Earth modificado por los autores b) Perspectiva del sector. Se observa la interacción con el perimetro y la propuesta brindada al barrio.

\section{MATERIALES Y MÉTODOS:}

Lainstalación fotovoltaica,está compuesta por un arreglo $\mathrm{FV}$ de 2,88 $\mathrm{kW}$. El espacio ocupado por los módulos fotovoltaicos se limita a la cubierta de una estructura del tipo "pérgola", con caída en una sola dirección, cubriendo una superficie total de $20 \mathrm{~m} 2$.

La instalación se compone de módulos FV del mismo modelo y potencia. Los módulos son de la Marca SolarWorld tipo policristalinos, con una potencia nominal de $240 \mathrm{Wp}$ y con unas medidas aproximadas de 1690 x $991 \mathrm{~mm}$.

Los 12 módulos se conectan eléctrica- mente en serie, con una inclinación de $20^{\circ}$ sobre la horizontal, y un azimut de $0^{\circ}$ (Norte Geográfico), resultando, pérdida por orientación estimada: $0 \%$ y pérdida por sombras circundantes: $<5 \%$ (debido a sombras de edificios que se proyectan desde el Oeste).

$\mathrm{El}$ inversor es monofásico de 2,8 kW de potencia nominal de la marca AEG.

El monitoreo se realiza a partir de una Celda de Silicio encapsulada, con características similares a las de los módulos, para medir radiación solar a través de la corriente de cortocircuito, $\mathrm{y}$ para medir Temperatura a través de la tensión de circuito abierto. 
Posee un Datalogger de 16 bits con 8 entradas analógicas para interpretar la señal de los sensores y comunicarlos y guardarlos en una PC.
En la Figura 2 a) se presenta el tablero de comando junto con el inversor para conexión ala redyenla Figura $2 \mathrm{~b}$ ) el denominado patio solar donde se encuentra la cubierta solar.

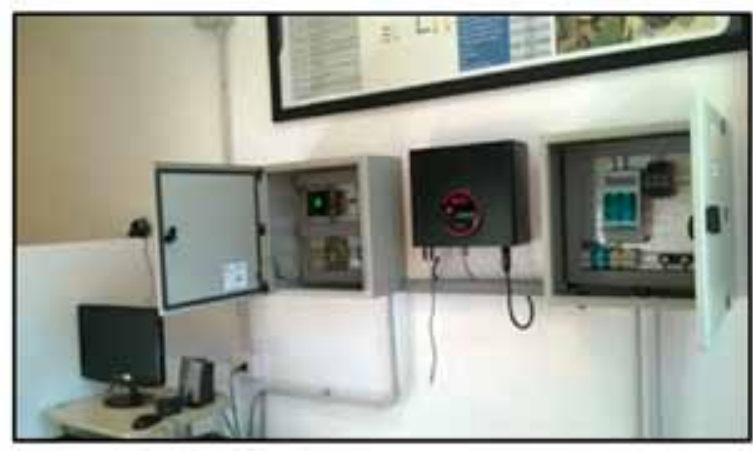

(a)

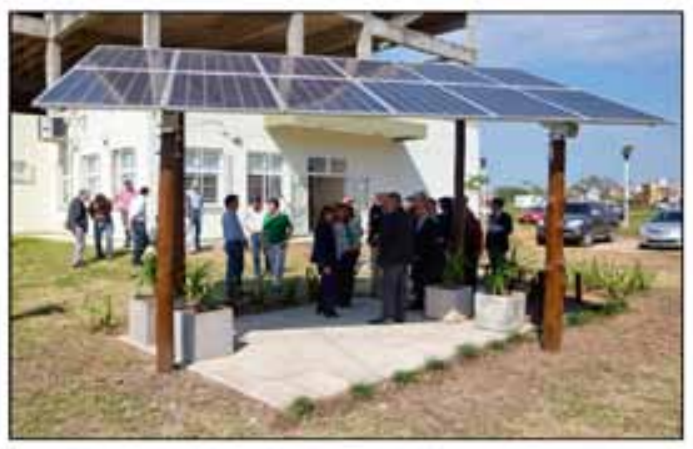

(b)

Figura 2 a) Tablero eléctrico del SFCR donde se observan la caja con protecciones de $\mathrm{AC}$, inversor, caja con protecciones $\mathrm{CC}$, datalogger y $\mathrm{PC}$ para almacenamiento de datos.

b) Foto del patio solar el dia de su inauguración.

En el SFCR los datos que son adquiridos, son: temperatura de inversor en ${ }^{\circ} \mathrm{C}$, tensión continua en volts, Corriente alterna en amperes, tensión alterna en volts, Frecuencia de salida en Hertz, Potencia alterna en watt, Energía total entregada en $\mathrm{kWh}$, y tiempo de entrega de potencia a la red en horas. La Irradiancia y temperatura de celda es medida utilizando unacelda de referencia desarrollada y calibrada en el GER-UNNE (Firman et al, 2014).

La dinámica del sistemas de monitoreo se basa en adquirir en forma paralela los datos que brinda el inversor por un lado, $y$ por otro las valores de variables climáticas. Losdatos que brinda el inversor son adquiriros a través de un software específico, y grabados de manera diaria, semanal, mensual y anual. $\mathrm{El}$ inversor comienza a tomar datos a partir de un cierto nivel de tensión en $\mathrm{CC}$, que se corresponde con un nivel de irradiancia y comienza a inyectar en la red (siempre que esta no tengas fallas como tensión baja o fuera de frecuencia). Deja de inyectar cuando el arreglo FV no puede entregar dicho nivel de tensión continua. Durante ese período, el software almacena los datos minuto a minuto. Para obtener esos datos, la interfaz del programa nos permite descargarlos en el intervalo de tiempo que deseemos, guardando una planilla de Excel del tipo CSV.

Los valores $d$ irradiancia y temperatura son medidos a través de la celda de referencia; la cual se conecta al datalogger, y este último a una PC. En la PC, se realiza la interpretación de los valores (en $\mathrm{mV}$ ) para traducirlos a irradiancia o temperatura, según corresponda. Esta tarea, la realiza un software desarrollado Visual Basic para ese fin, el cual además guarda los datos en archivos tipo txt. Los datos son grabados minuto a minuto e integrados y guardados en promedios horarios también. Se crean dos archivos por día, uno por minutos, y otro por horas.

Estos archivos se guardan en una ruta 
específica, de donde se puede acceder a los datos.

\section{RESULTADOS Y DISCUSIÓN:}

Se presenta seguidamente, la energía que fue inyectada efectivamente en la red, y la energía que recibió el arre- glo fotovoltaico en ese mismo período. Con esos valores se determinó el factor de desempeño o rendimiento, conocido en inglés como performance ratio (PR).

En la Tabla 1 se presentan los resultados de 7 meses de monitoreo

\begin{tabular}{|c|c|c|c|c|c|c|}
\hline Mes & Dias & Eac [KWh/mes] & $\begin{array}{c}\text { Horas } \\
\text { medidas [h] }\end{array}$ & Efv [KWh/m² dia] & $\begin{array}{c}\text { Eac específica } \\
{[\text { KWh/Kwp dia] }}\end{array}$ & $\begin{array}{c}\text { Factor de } \\
\text { rendimiento [\%] }]\end{array}$ \\
\hline Agosto & 31 & 369,7 & 315 & 5,31 & 4,14 & $78,03 \%$ \\
\hline Septiembre & 30 & 315,4 & 318 & 4,59 & 3,65 & $79,49 \%$ \\
\hline Octubre & 31 & 405,4 & 360 & 6,06 & 4,54 & $74,95 \%$ \\
\hline Noviembre & 30 & 431,5 & 379 & 6,83 & 4,99 & $73,13 \%$ \\
\hline Diciembre & 31 & 393,9 & 391 & 6,08 & 4,41 & $72,54 \%$ \\
\hline Enero & 31 & 404,5 & 383 & 6,42 & 4,53 & $70,59 \%$ \\
\hline Febrero & 28 & 380,2 & 333 & 6,66 & 4,71 & $70,79 \%$ \\
\hline
\end{tabular}

Tabla 1: Energía inyectada a la red, energía recibida por los módulos (irradiación), y factor de desempeño del sistema mensual.

La Eac [kWh/mes], es la energía que fue entregada por el inversor a la red. Ésta fue medida y almacenada por el software de comunicación que trae el inversor. Las horas medidas, son las horas totales por mes que el SFCR inyecto energía a la red. La $E f v[\mathrm{kWh} /$ día m2], es la energía total incidente sobre el plano del arreglo FV, medidas con la celda de referencia. Este último considerando su valor medio mensual es definida como Productividad de referencia o Reference Yield, YR, definido como la irradiación solar anual incidente en el plano del generador fotovoltaico, expresada en $\mathrm{kWh} / \mathrm{m} 2$, respecto de la radiación nominal de $1 \mathrm{~kW} / \mathrm{m}^{2}$ (Abella et al. CIEMAT).

La Eac específica también conocida como Horas Equivalentes de Sol, o Final Yield, YFen $[\mathrm{kWh} / \mathrm{kWp}$ día], es la energía media diaria inyectada, dividida por la potencia pico del arreglo fotovoltaico

Calculados estos valores es posible cal- cular el desempeño global del sistema. Según Abella et al (2010)el factor de desempeño como se define según la ecuación 1.

$$
P R=\frac{Y_{F}}{Y_{R}}
$$

Ec. 1

En la Figura3, se presentan dos escalas de gráficos de barras. Una de ellas muestra la energía mensual entregada a la red, y la otra el factor de desempeño que presentó el sistema. Ambas gráficas incluyen los meses de agosto de 2014 a febrero de 2015.

En la Figura 3 se observan los valores de desempeño del sistema y la energía mensual entregada. E1 SFCR tuvo un funcionamiento continuo sin inconvenientes, que resalta la confiabilidad que otorga.Se han medido algunas desconexiones del sistema asociados a cortes de energía y salida de frecuencia de la red, en los meses de octubre a diciembre. Esta es la razón por las cual en estos meses 


\section{Factor de Rendimiento (PR) mensual, y energía inyectada, del $08 / 2014$ al 02/2015}

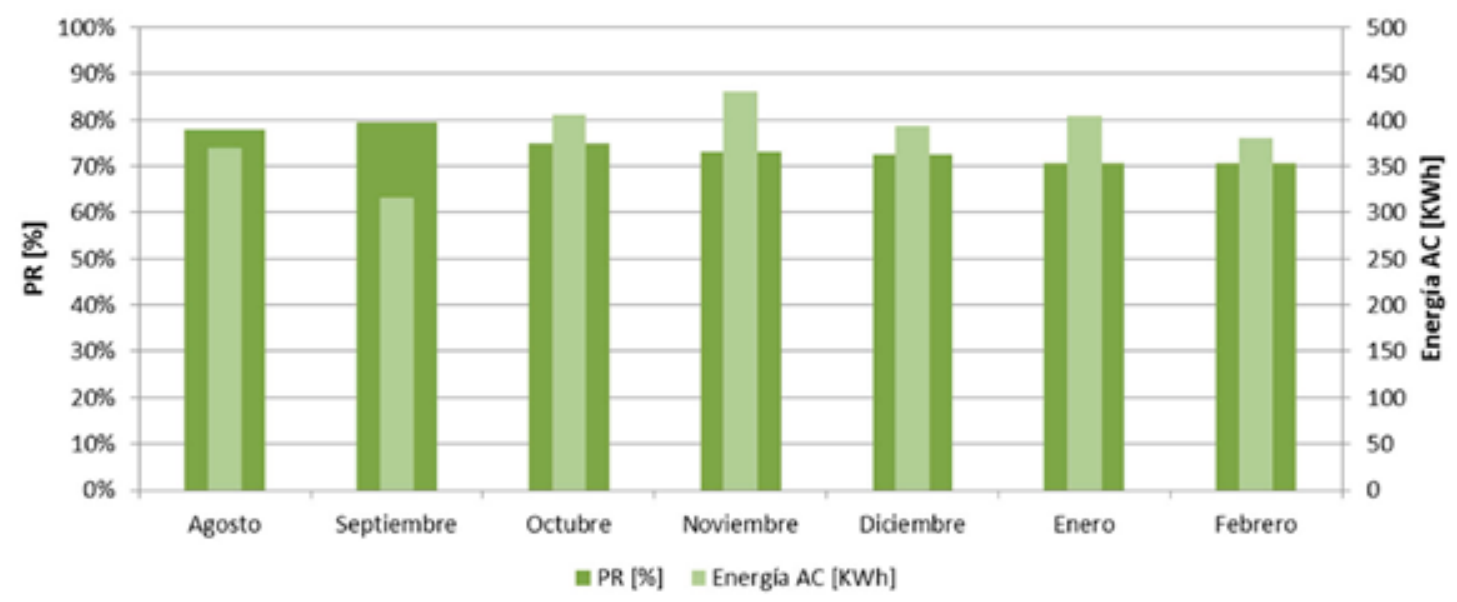

Figura 3. Energía AC y PR\% en siete meses de funcionamiento.

se observa una reducción del PR, además del factor de temperatura y efectos angulares.

Sobre la tasa de desempeño medida, se puede afirmar que se está frente a un sistema bien dimensionado, ya que las PR normales en SFV, rondan entre 70 y $75 \%$ (Salgado, 2010), y los valores mostrados hasta ahora, son entre un 70 y $80 \%$. Con lo que se evalúa una generación eléctrica aproximada de $4320 \mathrm{kWh} / \mathrm{año}$.

\section{CONCLUSIÓN}

Se ha proyectado, onstaldo y evaluado el comportamiento de un SFCR en el Campus de la Reforma de la UNNE. El correcto funcionamiento del sistema permite afirmar que la cubierta solar generará $4320 \mathrm{kWh} /$ año y será un aporte a la ciudad desde el punto de vista ambiental en al menos los siguientes aspectos:

- Será la primera experiencia de la ciudad de Resistencia en SFVCR.

- Generará energía que dis- minuirá el gasto del Campus de la UNNE sobre la red existente.

- Tendrá un efecto demostrativo, acercando la energía fotovoltaica a la sociedad.

- SeráunnuevoServicioAmbientalque el Campus Resistencia brindará a la ciudad.

- Permitirá la experimentación y el avance en el estudio de la energía solar.

Se espera que el Patio Urbano y en especial la "cubierta solar" tenga un importante "efecto demostrativo" tendiente a la difusión de la energía fotovoltaica (limpia, renovable y sustentable), mediante la incorporación del concepto de coexistencia de los sistemas tradicionales de generación y distribución de energía eléctrica, en un espacio útil para el esparcimiento y actividades académicas a cielo abierto, iniciativa que lo que representa un cambio conceptual.

De esta manera, se plantea una experiencia aplicada que contribuirá al conocimiento tecnológico necesario para im- 
pulsar un cambio de escala en el uso de la energía solar, mediante un trabajo interdisciplinario de distintas áreas de la
Universidad de las Facultades de Arquitectura, Ingeniería y Ciencias Exactas.

\section{REFERENCIAS}

Salgado F. 2010. "COMPENDIO DE ENERGÍA SOLAR: Fotovoltaica, Térmica y Termoeléctrica (Adaptado AL Código Técnico de le Edificación y al nuevo RITE)"

A. Firman, L. Zini, R. Sanchez, L. Vera. 2014. "Desarrollo y calibración de dispositivos fotovoltaicos para determinar el recurso solar utilizable por SFCR".

Abella, Miguel Alonso y Chenlo, Faustino. "Estimación de la energía generada por un sistema fotovoltaico conectado a red". CIEMAT. Madrid

Manual de Engenharia para Sistemas Fotovoltaicos. Organizadores: Joao Tavares Pinho. Marco Antonio Galdino. Grupo de trabalho de energía solar GTES - CEPEL - DTE CRESESB. Río de Janeiro - Marzo 2014.

CAMMESA (2016). Informe Anual 2015 República Argentina, Compañía Ad- ministradora del Mercado Mayorista Eléctrico S.A.. http://www.cammesa.com/linfoanu.nsf/MINFOANU?OpenFrameSet

IRESUD (2016). http://iresud.com.ar/

Laborde M.y Williams R. (2016). Energía Solar, Capítulo 1, "Energía Solar Fotovoltaica”, J. Plá, M.D. Perez, J.C. Durán. Academia Nacional de Ciencias Exactas, Físicas y Naturales, Buenos Aires. http:// www.ancefn.org.ar/biblioteca/libros.html

Munsell M. (2016). 5TrendsShapingthe 66GW Global Solar Market. http://www. greentechmedia.com/articles/read/5-Trends-Shaping-the-66-GW-Global-Solar-Market

REN21(2016)."Renewables 2016-Global Status Report”, informe generado por REN21-RenewableEnergyPolicy Network forthe 21st Century. www.ren21.net/REN21Activities/GlobalStatusReport.aspx 